\title{
VOLTERRA AND URYSOHN INTEGRAL EQUATIONS IN BANACH SPACES
}

\author{
DONAL O'REGAN \\ University College Galway, Department of Mathematics \\ Galway, IRELAND
}

(Received January, 1997; Revised June, 1997)

We use topological methods to present existence principles and theory for integral equations in Banach spaces. Tube.

Key words: Urysohn, Volterra, Equations in Abstract Spaces, Solution

AMS subject classifications: $45 \mathrm{G} 10,45 \mathrm{~N} 05$.

\section{Introduction}

In this paper we are concerned with Volterra and Urysohn equations in Banach spaces. The paper will be divided into two main sections. In Section 2 general existence principles are established for these equations. The technique relies on a nonlinear alternative of Leray-Schauder type [2]. Our results improve and extend results in [15]; in addition some of the results are new even in the finite dimensional setting. In Section 3 some applications are given. First an existence principle of Brezis-Browder type [1] is established for Hammerstein equations in Banach spaces. Also in Section 3 we give a notion of "solution tube" for singular second order differential equations in Hilbert spaces.

Throughout $E$ will be a Banach space with norm $\|\cdot\|$. We denote by $C([0, T], E)$ the space of continuous functions $u:[0, T] \rightarrow E$. Let $u:[0, T] \rightarrow E$ be a measurable function. By $\int_{0}^{T} u(s) d s$ we mean the Bochner integral of $u$, assuming it exists (see [16] for properties of the Bochner integral). The semi inner products ([7, 9]) on $E$ are defined by

$$
\begin{aligned}
& \langle x, y\rangle_{+}=\|x\| \lim _{t \rightarrow 0^{+}} \frac{\|x+t y\|-\|x\|}{t} \\
& \langle x, y\rangle_{-}=\|x\| \lim _{t \rightarrow 0^{-}}-\frac{\|x+t y\|-\|x\|}{t} .
\end{aligned}
$$

Let $\Omega_{E}$ be the bounded subsets of $E$. Let $X \in \Omega_{E}$. The diameter of $X$ is defined by

$$
\operatorname{diam}(X)=\sup \{\|x-y\|: x, y \in X\}
$$


The Kuratowskii measure of noncompactness is the map $\alpha: \Omega_{E} \rightarrow[0, \infty]$ defined by

$$
\alpha(X)=\inf \left\{\epsilon>0: X \subseteq \bigcup_{i=1}^{n} X_{i} \text { and } \operatorname{diam}\left(X_{i}\right) \leq \epsilon\right\}
$$

Theorem 1.1: Let $T>0$ and $E$ be a Banach space.

(i) Let $A \subseteq C([0, T], E)$ be bounded. Then

$$
\sup _{t \in[0, T]} \alpha(A(t)) \leq \alpha(A[0, T]) \leq 2 \alpha(A)
$$

where $A(t)=\{\phi(t): \phi \in A\}$ and $A[0, T]=\bigcup_{t \in[0, T]}\{\phi(t): \phi \in A\}$.

(ii) Let $A \subseteq C([0, T], E)$ be bounded and equicontinuous. Then

$$
\alpha(A)=\sup _{t \in[0, T]} \alpha(A(t))=\alpha(A[0, T])
$$

Proof: (i) For each $t \in[0, T]$ we have $A(t) \subseteq A[0, T]$ and so $\alpha(A(t)) \leq$ $\alpha(A[0, T])$ which gives

$$
\sup _{t \in[0, T]} \alpha(A(t)) \leq \alpha(A[0, T])
$$

The other inequality follows from the ideas in [7, page 24].

(ii) The result follows from [7,9].

Let $E_{1}$ and $E_{2}$ be two Banach spaces and let $F: Y \subseteq E_{1} \rightarrow E_{2}$ be continuous and map bounded sets into bounded sets. We call such an $F$ a $\alpha$-Lipschitzian map if there is a constant $k \geq 0$ with $\alpha(F(X)) \leq k \alpha(X)$ for all bounded sets $X \subseteq Y$. We also say $F$ is a Darbo map if $F$ is $\alpha$-Lipschitzian with $k<1$. Next we state a fixed point result due to Sadovskii [2].

Theorem 1.2: Let $K$ be a closed, convex subset of a Banach space $B$ and let $N: K \rightarrow K$ be a bounded Darbo map. Then $N$ has a fixed point in $K$.

We also have the following nonlinear alternative of Leray-Schauder type for Darbo maps [2].

Theorem 1.3: Let $K$ be a closed, convex subset of a Banach space B. Assume $U$ is a relatively open subset of $K$ with $0 \in U, N(\bar{U})$ bounded and $N: \bar{U} \rightarrow K$ a Darbo map. Then either

(A1) $N$ has a fixed point in $\bar{U}$; or

(A2) there is $a \in \in U$ and $\lambda \in(0,1)$ with $u=\lambda N u$.

\section{Existence Principles}

In this section we establish existence principle for the Volterra integral equation

$$
y(t)=h(t)+\int_{0}^{t} K(t, s, y(s)) d s, \quad t \in[0, T]
$$

and the Urysohn integral equation

$$
y(t)=h(t)+\int_{0}^{T} K(t, s, y(s)) d s, \quad t \in[0, T] .
$$


We will look for solutions in $C([0, T], E)$; here $E$ is a Banach space with norm $\|\cdot\|$. The ideas involved in establishing existence principles for both (2.1) and (2.2) are essentially the same; as a result we will examine (2.1) in detail and just state the results for (2.2).

Theorem 2.1: Let $K:[0, T] \times[0, t] \times E \rightarrow E$. Suppose

$$
\left\{\begin{array}{l}
\text { there exists a constant } \gamma \geq 0 \text { with } \alpha(K([0, T] \times[0, t] \times \Omega)) \leq \gamma \alpha(\Omega) \\
\text { for each bounded set } \Omega \subseteq E
\end{array}\right.
$$

$$
2 \gamma T<1
$$

$K:[0, T] \times[0, t] \times E \rightarrow E$ is $L^{1}$-Carathéodory uniformly in $t$; by this we mean

(i) for each $t \in[0, T]$, the map $u \mapsto K_{t}(s, u)$ is continuous for almost all $s \in[0, t]$

(note for each $t \in[0, T], K_{t}:[0, t] \times E \rightarrow E$ is defined by $K_{t}(s, u)=K(t, s, u)$ )

(ii) for each $t \in[0, T]$, the map $s \mapsto K_{t}(s, u)$ is measurable for all $u \in E$

(iii) for each $t \in[0, T]$ and for each $r>0$ there exists $h_{t, r} \in L^{1}([0, T], \mathbf{R})$

such that $\|u\| \leq r$ implies $\left\|K_{t}(s, u)\right\| \leq h_{t, r}(s)$ for almost all $s \in[0, t]$

(iv) for each $r>0$ there exists $h_{r} \in L^{1}([0, T], \mathbf{R})$ and $\alpha>0$ such that for $x<z$ in $[0, T], \int_{x}^{z} h_{z, r}(s) d s \leq\left(\int_{x}^{z} h_{r}(s) d s\right)^{\alpha}$

$$
\left\{\begin{array}{l}
\text { for any } r>0 \text { and any } z, t \in[0, T] \text { we have that } \\
\int_{0}^{t_{1}} \sup _{\|u\| \leq r}\|K(z, s, u)-K(t, s, u)\| d s \rightarrow 0 \text { as } z \rightarrow t \\
\text { where } t_{1}=\min \{t, z\}
\end{array}\right.
$$

and

$$
h \in C([0, T], E)
$$

hold. In addition assume that there is a constant $M_{0}$, independent of $\lambda$, with

$$
\|y\|_{0}=\sup _{[0, T]}\|y(t)\| \neq M_{0}
$$

for any solution $y \in C([0, T], E)$ to

$$
y(t)=\lambda\left(h(t)+\int_{0}^{t} K(t, s, y(s)) d s\right), \quad t \in[0, T]
$$

for each $\lambda \in[0,1]$. Then $(2.1)$ has a solution in $C([0, T], E)$.

Remark: Theorem 2.1 improves a result in [15, Section 3]. 
Proof: Define the operator $N: C([0, T], E) \rightarrow C([0, T], E)$ by

$$
N y(t)=h(t)+\int_{0}^{t} K(t, s, y(s)) d s .
$$

Now $(2.8)_{\lambda}$ is equivalent to the fixed point problem $y=\lambda N y$. We would like to apply Theorem 1.3. First we show $N: C([0, T], E) \rightarrow C([0, T], E)$ is continuous. To see this, let $u_{n} \rightarrow u$ in $C([0, T], E)$. Then there exists $r>0$ with $\left\|u_{n}(s)\right\| \leq r$ and $\|u(s)\| \leq r$ for all $s \in[0, T]$. Also for each $t \in[0, T]$ there exists $h_{t, r} \in L^{1}([0, T], \mathbf{R})$ with

$$
\|K(t, s, v)\| \leq h_{t, r}(s) \text { for a.e. } s \in[0, T] \text { and all }\|v\| \leq r .
$$

Now from (2.5) we have for each $t \in[0, T]$ that

$$
K\left(t, s, u_{n}(s)\right) \rightarrow K(t, s, u(s)) \text { for almost all } s \in[0, T] .
$$

This together with the Lebesgue dominated convergence theorem yields $N u_{n}(t) \rightarrow$ $N u(t)$ pointwise on $[0, T]$. Now (2.6) guarantees that the convergence is uniform (i.e., the argument below will show that for any $\epsilon>0$ there exists $\delta>0$ such that for $t, t^{\prime} \in[0, T]$ with $\left|t-t^{\prime}\right|<\delta$ we have $\left\|N u_{n}\left(t^{\prime}\right)-N u_{n}(t)\right\|<\epsilon$ for all $n$ and $\left.\left\|N u\left(t^{\prime}\right)-N u(t)\right\|<\epsilon\right)$. Hence $N u \in C([0, T], E)$ and $N$ is continuous.

Next, let $\Omega$ be a bounded subset of $C([0, T], E)$. We first claim that $N \Omega$ is bounded and equicontinuous on $[0, T]$. Then there exists $r>0$ with $\|u(s)\| \leq r$ for all $s \in[0, T]$ and $u \in \Omega$. Also there exists $h_{t, r}$ and $h_{r}$ as in (2.5). Now $N \Omega$ is bounded since for $t \in[0, T]$ and $u \in \Omega$ we have

$$
\begin{aligned}
\|N u(t)\| & \leq \sup _{[0, T]}\|h(t)\|+\int_{0}^{t} \sup _{\|v\| \leq r}\|K(t, s, v)\| d s \\
& \leq \sup _{[0, T]}\|h(t)\|+\left(\int_{0}^{T} h_{r}(w) d w\right)^{\alpha} .
\end{aligned}
$$

Also, for $t, t^{\prime} \in[0, T]$ with $t^{\prime}>t$ and $u \in \Omega$, we have

$$
\begin{aligned}
\left\|N u\left(t^{\prime}\right)-N u(t)\right\| & \leq\left\|h\left(t^{\prime}\right)-h(t)\right\|+\int_{0}^{t} \sup _{\|v\| \leq r}\left\|K\left(t^{\prime}, s, v\right)-K(t, s, v)\right\| d s \\
& +\int_{t}^{t^{\prime}} \sup _{\|v\| \leq r}\left\|K\left(t^{\prime}, s, v\right)\right\| d s \\
& \leq\left\|h\left(t^{\prime}\right)-h(t)\right\|+\int_{0}^{t} \sup _{\|v\| \leq r}\left\|K\left(t^{\prime}, s, v\right)-K(t, s, v)\right\| d s \\
& +\left(\int_{t}^{t^{\prime}} h_{r}(s) d s\right)^{\alpha} .
\end{aligned}
$$


Consequently $N \Omega$ is equicontinuous on $[0, T]$. We now show

For $t \in[0, T]$, we have

$$
\alpha(N \Omega) \leq 2 \gamma T \alpha(\Omega)
$$

$$
\begin{aligned}
\alpha(N \Omega(t)) & =\alpha\left(\left\{h(t)+\int_{0}^{t} K(t, s, u(s)) d s: u \in \Omega\right\}\right) \\
& \leq \alpha(t \overline{c o}\{K(t, s, u(s)): y \in \Omega, s \in[0, t]\}) \\
& =t \alpha(\{K(t, s, u(s)): y \in \Omega, s \in[0, t]\}) \\
& \leq T \alpha(K([0, T] \times[0, t] \times \Omega[0, t])) \\
& \leq T \gamma \alpha(\Omega[0, t])
\end{aligned}
$$

where $\Omega[0, t]=\bigcup_{s \in[0, t]}\{\phi(s): \phi \in \Omega\}$. Theorem $1.1(i)$ implies

$$
\alpha(N \Omega(t)) \leq 2 \gamma T \alpha(\Omega)
$$

In addition since $N \Omega$ is bounded and equicontinuous on $[0, T]$ we have from Theorem 1.1 (ii) that

$$
\alpha(N \Omega)=\sup _{t \in[0, T]} \alpha(N \Omega(t))
$$

and this together with (2.10) implies that (2.9) is true. Let

$$
U=\left\{u \in C([0, T], E):\|u\|_{0}<M_{0}\right\}, B=K=C([0, T], E) .
$$

Now Theorem 1.3 (notice $(A 2)$ cannot occur) implies that (2.1) has a solution in $\bar{U}$. A special case of $(2.1)$ is

$$
y(t)=h(t)+\int_{0}^{t} k(t, s) f(s, y(s)) d s, t \in[0, T]
$$

where $k$ takes values in $\mathbf{R}$.

Theorem 2.2: Let $k:[0, T] \times[0, t] \rightarrow \mathbf{R}$ and $K(t, s, u)=k(t, s) f(s, u)$. Assume (2.3), (2.4) and (2.7) hold. Also suppose

$f:[0, T] \times E \rightarrow E$ is a $L^{q}$-Carathéodory function (here $q>1$ is a constant); by this we mean

(i) the map $t \mapsto f(t, z)$ is measurable for all $z \in E$

(ii) the map $z \mapsto f(t, z)$ is continuous for almost all $t \in[0, T]$

(iii) for each $r>0$ there exists $\mu_{r} \in L^{q}([0, T], \mathbf{R})$ such that $\|z\| \leq r$ implies $\|f(t, z)\| \leq \mu_{r}(t)$ for almost all $t \in[0, T]$ 
$\left\{\begin{array}{l}k_{t}(s) \in L^{p}([0, t], \mathbf{R}) \text { for each } t \in[0, t] ; \text { here } \frac{1}{p}+\frac{1}{q}=1 \text { and for } \\ \text { each } t \in[0, T], k_{t}:[0, t] \rightarrow \mathbf{R} \text { is defined by } k_{t}(s)=k(t, s)\end{array}\right.$

and

$$
\left\{\begin{array}{l}
\text { for any } t_{1}, t_{2} \in[0, T] \text { we have that } \\
\int_{0}^{t_{3}}\left|k_{t_{1}}(s)-k_{t_{2}}(s)\right|^{p} d s \rightarrow 0 \text { as } t_{1} \rightarrow t_{2} \\
\text { where } t_{3}=\min \left\{t_{1}, t_{2}\right\}
\end{array}\right.
$$

are satisfied. In addition, assume there is a constant $M_{0}$, independent of $\lambda$, with $\|y\|_{0} \neq M_{0}$ for any solution $y \in C([0, T], E)$ to

$$
y(t)=\lambda\left(h(t)+\int_{0}^{t} k(t, s) f(s, y(s)) d s\right), t \in[0, T]
$$

for each $\lambda \in[0,1]$. Then $(2.11)$ has a solution in $C([0, T], E)$.

Remark: One could also discuss the case $q=1$ in Theorem 2.2 .

Proof: The results follows from Theorem 2.1 once we show (2.5) and (2.6) are true. Notice first for any $r>0$ and any $u \in E$ with $\|u\| \leq r$ there exists $\mu_{r} \in L^{q}([0, T], \mathbf{R})$ with $\|f(s, u)\| \leq \mu_{r}(s)$ for almost all $s \in[0, T]$. Then for each $t \in[0, T]$ and $\|u\| \leq r$ we have

$$
\|K(t, s, u)\| \leq|k(t, s)| \mu_{r}(s) \equiv h_{t, r}(s) \text { for almost all } s \in[0, T] .
$$

By Hölder's inequality for $x, z \in[0, T]$ with $x<z$, we have

and so

$$
\begin{aligned}
\int_{x}^{z} h_{z, r}(s) d s & \leq\left(\int_{0}^{z}\left|k_{z}(s)\right|^{p} d s\right)^{\frac{1}{p}}\left(\int_{x}^{z} \mu_{r}^{q}(s) d s\right)^{\frac{1}{q}} \\
& \leq \max _{z \in[0, T]}\left(\int_{0}^{z}\left|k_{z}(s)\right|^{p} d s\right)^{\frac{1}{p}}\left(\int_{x}^{z} \mu_{r}^{q}(s) d s\right)^{\frac{1}{q}}
\end{aligned}
$$

where

$$
\int_{x}^{z} h_{z, r}(s) d s \leq\left(\int_{x}^{z} h_{r}(s) d s\right)^{\alpha}
$$

$$
\alpha=\frac{1}{q}>0 \text { and } h_{r}=\left[c \mu_{r}\right]^{q} \text { with } c=\max _{z \in[0, T]}\left(\int_{0}^{z}\left|k_{z}(s)\right|^{p} d s\right)^{\frac{1}{p}} \text {. }
$$

Consequently (2.5) is true. Finally (2.6) follows since if $t>z$, we have

$$
\int_{0}^{z} \sup _{\|u\| \leq r}\|K(z, s, u)-K(t, s, u)\| d s=\int_{0}^{z}|k(z, s)-k(t, s)| \sup _{\|u\| \leq r}\|f(s, u)\| d s
$$




$$
\leq\left(\int_{0}^{z}\left|k_{z}(s)-k_{t}(s)\right|^{p} d s\right)^{\frac{1}{p}}\left(\int_{0}^{T} \mu_{r}^{q}(s) d s\right)^{\frac{1}{q}} \rightarrow 0 \text { as } z \rightarrow t .
$$

Essentially the same reasoning as in Theorem 2.1 establishes the following existence principle for the Urysohn integral equation (2.2).

Theorem 2.3: Let $K:[0, T] \times[0, T] \times E \rightarrow E$. Suppose

$\left\{\begin{array}{l}\text { there exists a constant } \gamma \geq 0 \text { with } \alpha(K([0, T] \times[0, T] \times \Omega)) \leq \gamma \alpha(\Omega) \\ \text { for each bounded set } \Omega \subseteq E\end{array}\right.$

$$
2 \gamma T<1
$$

$K:[0, T] \times[0, T] \times E \rightarrow E$ is $L^{1}$-Carathéodory in $t$;

by this we mean for each $t \in[0, T]$;

(i) the map $u \mapsto K_{t}(s, u)$ is continuous for almost all $s \in[0, T]$

(ii) the map $s \mapsto K_{t}(s, u)$ is measurable for all $u \in E$

(iii) for each $r>0$ there exists $h_{t, r} \in L^{1}([0, T], \mathbf{R})$ such that

$\|u\| \leq r$ implies $\left\|K_{t}(s, u)\right\| \leq h_{t, r}(s)$ for almost all $s \in[0, T]$

$\left\{\begin{array}{l}K \text { is integrably bounded in } t \text { i.e. } \sup _{t \in[0, T]} \int_{0}^{T} h_{t, r}(s) d s<\infty \\ \text { where } h_{t, r}(s) \text { is as in }(2.18)(i i i)\end{array}\right.$

and

$$
\lim _{z \rightarrow t} \int_{0}^{T} \sup _{\|u\| \leq r}\|K(z, s, u)-K(t, s, u)\| d s=0
$$

$$
h \in C([0, T], E)
$$

hold. In addition assume there is a constant $M_{0}$, independent of $\lambda$, with $\|y\|_{0} \neq M_{0}$ for any solution $y \in C([0, T], E)$ to

$$
y(t)=\lambda\left(h(t)+\int_{0}^{T} K(t, s, y(s)) d s\right), \quad t \in[0, T]
$$

for each $\lambda \in[0,1]$. Then $(2.2)$ has a solution in $C([0, T], E)$.

Theorem 2.3 immediately yields the following result for the Hammerstein integral equation 


$$
y(t)=h(t)+\int_{0}^{T} k(t, s) f(s, y(s)) d s, t \in[0, T]
$$

Theorem 2.4: Let $k[0, T] \times[0, T] \rightarrow \mathbf{R}$ and $K(t, s, u)=k(t, s) f(s, u)$. Assume (2.16), (2.17) and (2.21) hold. Also suppose

$f:[0, T] \times E \rightarrow E$ is a $L^{q}$-Carathéodory function (here $q>1$ is a constant)

$$
k_{t}(s) \in L^{p}([0, T], \mathbf{R}) \text { for each } t \in[0, T] ; \text { here } \frac{1}{p}+\frac{1}{q}=1
$$

and

$$
\text { the map } t \mapsto k_{t} \text { is continuous from }[0, T] \text { to } L^{p}([0, T], \mathbf{R})
$$

are satisfied. In addition assume there is a constant $M_{0}$, independent of $\lambda$, with $\|y\|_{0} \neq M_{0}$ for any solution $y \in C([0, T], E)$ to

$$
y(t)=\lambda\left(h(t)+\int_{0}^{T} k(t, s) f(s, y(s)) d s\right), \quad t \in[0, T]
$$

for each $\lambda \in[0,1]$. Then $(2.23)$ has a solution in $C([0, T], E)$.

\section{Applications}

In this section we use the existence principles of Section 2 to establish existence theory for various integral equations. We begin by discussing the Hammerstein equation

$$
y(t)=h(t)+\int_{0}^{T} k(t, s) f(s, y(s)) d s, \quad t \in[0, T] .
$$

Remark: An existence theory of "superlinear" type could easily be developed for (3.1) (or indeed the Urysohn integral equation (2.2)) using the ideas in [13]; however since the reasoning involved is essentially the same, we as a result will not include results of this type here.

We first establish a result of Brezis-Browder type [1] for (3.1).

Theorem 3.1: Let $k:[0, T] \times[0, T] \rightarrow \mathbf{R}$ and $K(t, s, u)=k(t, s) f(s, u)$ and assume (2.16), (2.17), (2.21), (2.24), (2.25) and (2.26) hold. In addition suppose

$$
\left\{\begin{array}{l}
\text { there exists } R>0 \text { and a constant } a_{0}>0 \text { with } \\
\langle f(t, y), y\rangle_{+} \geq a_{0}\|y\|\|f(t, y)\| \text { for }\|y\| \geq R \text { and a.e. } t \in[0, T]
\end{array}\right.
$$

$\left\{\begin{array}{l}\text { there exists constants } \eta>0, \gamma \text { with } \gamma \geq q-1 \text { and a function } \phi \in L^{p}([0, T], \mathbf{R}) \\ \text { with }\|y\| \geq \eta\|f(t, y)\|^{\gamma}+\phi(t) \text { for }\|y\| \geq R \text { and a.e. } t \in[0, T]\end{array}\right.$ 
and

$$
\left\{\begin{array}{l}
\text { there exists a constant } A_{0} \geq 0 \text { with for any } u \in C([0, T], E) \\
\int_{0}^{T}\left\langle f(t, u(t)), \int_{0}^{T} k(t, s) f(s, u(s)) d s\right\rangle_{+} d t \leq A_{0}
\end{array}\right.
$$

are satisfied. Then (3.1) has a solution in $C([0, T], E)$.

Proof: Let $y$ be a solution of $(2.27)_{\lambda}$. We have (recall $\langle x, y+z\rangle_{+} \leq\langle x, y\rangle_{+}+$ $\langle x, z\rangle+$ where $x, y, z \in B$, a Banach space),

$$
\begin{aligned}
\int_{0}^{T}\langle f(t, y(t)), y(t)\rangle_{+} d t & \leq \int_{0}^{T}\langle f(t, y(t)), h(t)\rangle_{+} d t \\
& +\lambda \int_{0}^{T}\left\langle f(t, y(t)), \int_{0}^{T} k(t, s) f(s, y(s)) d s\right\rangle_{+} d t
\end{aligned}
$$

and so

$$
\int_{I}\langle f(t, y(t)), y(t)\rangle_{+} d t \leq \int_{0}^{T}\|h(t)\|\|f(t, y(t))\| d t+\left|A_{0}\right| .
$$

Let

$$
I=\{t \in[0, T]:\|y(t)\| \geq R\} \text { and } J=\{t \in[0, T]:\|y(t)\|<R\}
$$

Notice

$$
\begin{aligned}
\int_{0}^{T}\langle f(t, y(t)), y(t)\rangle_{+} d t & \geq a_{0} \int_{I}\|y(t)\|\|f(t, y(t))\| d t \\
& \geq \eta a_{0} \int_{I}\|f(t, y(t))\|^{\gamma+1} d t+a_{0} \int_{I} \phi(t)\|f(t, y(t))\| d t .
\end{aligned}
$$

Put this into (3.5) to obtain

$$
\begin{aligned}
\eta a_{0} \int_{I} \| f(t, y(t)) & \|^{\gamma+1} d t \\
& \leq a_{0} \int_{I}|\phi(t)|\|f(t, y(t))\| d t+\int_{J}\|y(t)\|\|f(t, y(t))\| d t \\
& +\int_{0}^{T}\|h(t)\|\|f(t, y(t))\| d t+\left|A_{0}\right| .
\end{aligned}
$$

Since $f$ is $L^{q}$-Carathéodory there exists $\mu_{R} \in L^{q}([0, T], \mathbf{R})$ such that $\|u\| \leq R$ implies $\|f(t, u)\| \leq \mu_{R}(t)$ for a.e. $t \in[0, T]$. Thus

$$
\begin{aligned}
\int_{J}\|y(t)\|\|f(t, y(t))\| d t+ & \int_{J}\|h(t)\|\|f(t, y(t))\| d t+\left|A_{0}\right| \\
& \leq R \int_{0}^{T} \mu_{R}(t) d t+\int_{0}^{T}\|h(t)\| \mu_{R}(t) d t+\left|A_{0}\right| \equiv A_{1}
\end{aligned}
$$


and so

$$
\begin{aligned}
& \eta \alpha_{0} \int_{I}\|f(t, y(t))\|^{\gamma+1} d t \\
& \quad \leq a_{0} \int_{I}|\phi(t)|\|f(t, y(t))\| d t+\int_{I}\|h(t)\|\|f(t, y(t))\| d t+A_{1} .
\end{aligned}
$$

Apply Hölder's inequality to obtain

$$
\begin{aligned}
& \eta a_{0} \int_{I}\|f(t, y(t))\|^{\gamma+1} d t \\
& \leq a_{0} T^{\frac{p \gamma-(\gamma+1)}{p(\gamma+1)}}\left(\int_{0}^{T}|\phi(s)|^{p} d s\right)^{\frac{1}{p}}\left(\int_{I}\|f(t, y(t))\|^{\gamma+1} d t\right)^{\frac{1}{\gamma+1}} \\
& +\left(\int_{0}^{T}\|h(t)\|^{\frac{\gamma+1}{\gamma}} d t\right)^{\frac{\gamma}{\gamma+1}}\left(\int_{I}\|f(t, y(t))\|^{\gamma+1} d t\right)^{\frac{1}{\gamma+1}} .
\end{aligned}
$$

There exists a constant $A_{2}$ with

$$
\int_{I}\|f(t, y(t))\|^{\gamma+1} d t \leq A_{2}
$$

Returning to $(2.27)_{\lambda}$ we have for $t \in[0, T]$ that

$$
\begin{aligned}
\|y(t)\| \quad & \leq \sup _{[0, T]}\|h(t)\|+\int_{J}\|k(t, s) f(s, y(s))\| d s+\int_{I}\|k(t, s) f(s, y(s))\| d s \\
& \leq \sup _{[0, T]}\|h(t)\|+\sup _{t \in[0, T]}\left(\int_{0}^{T}|k(t, s)|^{p} d s\right)^{\frac{1}{p}}\left(\int_{0}^{T} \mu_{R}(s) d s\right)^{\frac{1}{q}} \\
& \quad \frac{T^{\frac{p \gamma-(\gamma+1)}{p(\gamma+1)}} \sup _{t \in[0, T]}\left(\int_{0}^{T}|k(t, s)|^{p} d s\right)^{\frac{1}{p}} A_{2}^{\frac{1}{\gamma+1}} \equiv M_{0}}{}
\end{aligned}
$$

and so

$$
\sup _{[0, T]}\|y(t)\| \leq M_{0}
$$

for any solution $y$ to $(2.27)_{\lambda}$. The result follows from Theorem 2.4.

Essentially the same reasoning as in Theorem 3.1 establishes the following existence result for the Volterra equation

$$
y(t)=h(t)+\int_{0}^{t} k(t, s) f(s, y(s)) d s, t \in[0, T] .
$$


Theorem 3.2: Let $k:[0, T] \times[0, t] \rightarrow \mathbf{R}$ and $K(t, s, u)=k(t, s) f(s, u)$ and assume (2.3), (2.4), (2.7), (2.12), (2.13), (2.14), (3.2) and (3.3) hold. In addition, suppose

$$
\left\{\begin{array}{l}
\text { there exists a constant } A_{0} \geq 0 \text { with for any } u \in C([0, T], E), \\
\int_{0}^{T}\left\langle f(t, u(t)), \int_{0}^{t} k(t, s) f(s, u(s)) d s\right\rangle_{+} d t \leq A_{0}
\end{array}\right.
$$

is satisfied. Then $(3.6)$ has a solution in $C([0, T], E)$.

Remark: Existence theory of "growth type" could also easily be developed for (3.6) (or indeed (2.1)) using the ideas in [12].

For our next application we will examine the abstract Dirichlet boundary value problem

$$
\left\{\begin{array}{l}
y^{\prime \prime}+f(t, y)=0 \text { a.e. on }[0,1] \\
y(0)=y(1)=0
\end{array}\right.
$$

here $y:[0,1] \rightarrow H$ where $H$ is a Hilbert space with inner product $\langle\cdot, \cdot\rangle$. We give a notion of "solution tube" for such problems in the Hilbert space setting. Our theory was motivated by ideas in $[4,5]$. We will assume that $f:[0,1] \times H \rightarrow H$ is a $L_{l o c}^{1}$ Carathéodory function. By this we mean

(i) the map $t \mapsto f(t, z)$ is measurable for all $z \in E$;

(ii) the map $z \mapsto f(t, z)$ is continuous for almost all $t \in[0,1]$;

(iii) for any $r>0$ there exists $h_{r} \in L_{l o c}^{1}(0,1)$ with $\|f(t, z)\| \leq h_{r}(t)$ for almost all $t \in[0,1]$ and all $\|z\| \leq r$; also $\int_{0}^{1} x(1-x) h_{r}(x) d x<\infty$ with $\lim _{t \rightarrow 0^{+}}+$ $t^{2}(1-t) h_{r}(t)=0$ if $\int_{0}^{1}(1-x) h_{r}(x) d x=\infty$ and $\lim _{t \rightarrow 1}-t(1-t)^{2} h_{r}(t)=0$ if $\int_{0}^{1} x h_{r}(x) d x=\infty$.

Remark: It is worth remarking that other boundary data (homogeneous and nonhomogeneous) could also be considered here. However in our opinion (3.8) is the "most difficult" to examine (i.e., the "most singular") and as a result we will concentrate our study on (3.8).

By a solution to (3.8) we mean a function $y \in A C([0,1], H) \cap C^{1}([0,1], H)$ with $y^{\prime} \in A C_{l o c}((0,1), H)$ which satisfies the differential equation in (3.8) almost everywhere, and the stated boundary data. One can check (see $[10,14])$ that solving $(3.8)$ is equivalent to finding a function $y \in C([0,1], H)$ to

where

$$
y(t)=\int_{0}^{1} k(t, s) f(s, y(s)) d s, \quad t \in[0,1]
$$

$$
k(t, s)= \begin{cases}(1-t) s, & 0 \leq s \leq t \\ (1-s) t, & t \leq s \leq 1\end{cases}
$$

Remark: Notice $h_{r}$ in (iii) above is not necessarily in $L^{1}[0,1]$.

Theorem 3.3: Let $K(t, s, u)=k(t, s) f(s, u)$ and suppose the following conditions are satisfied: 
$\left\{\begin{array}{l}\text { there exists a constant } 0 \leq 2 \gamma<1 \text { with } \alpha(K([0,1] \times[0,1] \times \Omega)) \leq \gamma \alpha(\Omega) \\ \text { for each bounded set } \Omega \subseteq H\end{array}\right.$

$$
f:[0,1] \times H \rightarrow H \text { is a } L_{l o c}^{1} \text {-Carathéodory function }
$$

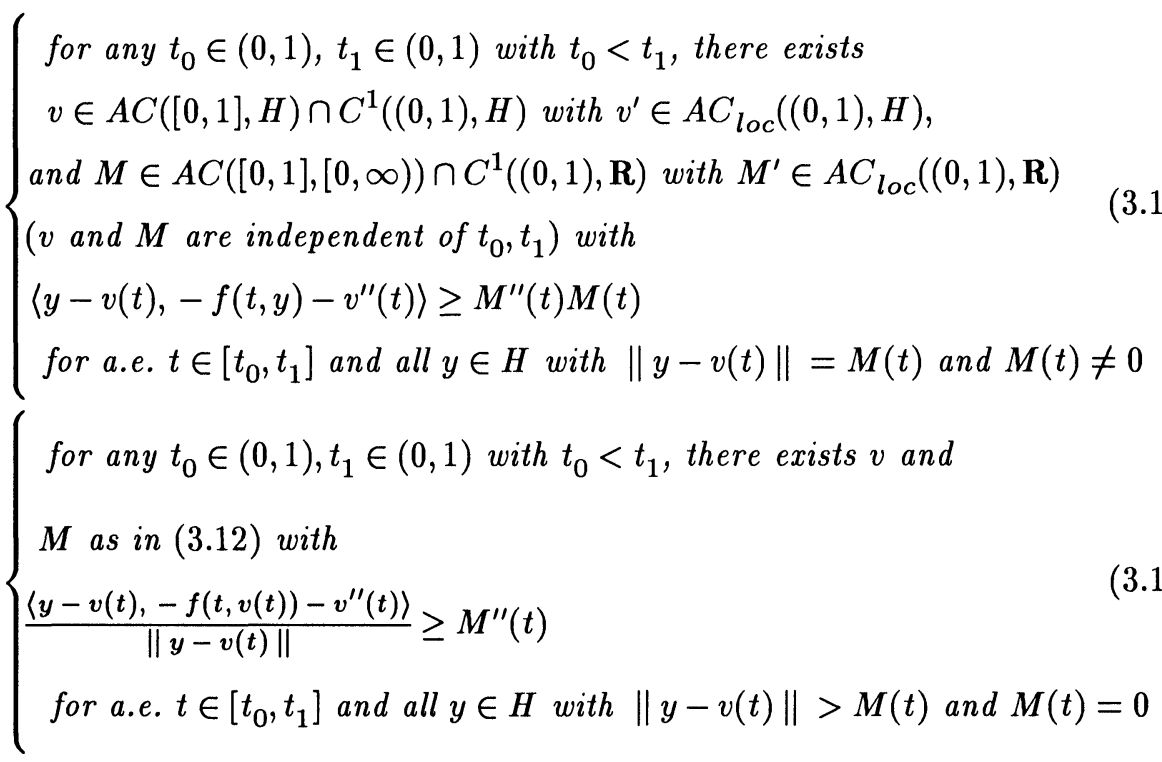

and

$$
\|v(0)\| \leq M(0) \text { and }\|v(1)\| \leq M(1) .
$$

Then (3.8) has a solution $y$ with $\|y(t)-v(t)\| \leq M(t)$ for all $t \in[0,1]$.

Proof: Consider the problem

where

$$
y(t)=\int_{0}^{1} k(t, s) f(s, p(s, y(s))) d s, t \in[0,1]
$$

$$
p(t, y)=\min \left\{1, \frac{M(t)}{\|y-v(t)\|}\right\} y+\left(1-\min \left\{1, \frac{M(t)}{\|y-v(t)\|}\right\}\right) v(t)
$$

i.e.

$$
p(t, y)=\left\{\begin{array}{cc}
y, & \text { if }\|y-v(t)\| \leq M(t) \\
M(t) \frac{y-v(t)}{\|y-v(t)\|}+v(t), & \text { if }\|y-v(t)\|>M(t)
\end{array}\right.
$$

is the radial retraction of $H$ onto $\{y$ : $\|y-v(t)\| \leq M(t)\}$. We now show (3.15) has solution in $C([0,1], H)$ by applying Theorem 1.2 . Define the operator $N$ : $C([0,1], H) \rightarrow C([0,1], H)$ by

$$
N y(t)=\int_{0}^{1} k(t, s) f(s, p(s, y(s))) d s .
$$

Let $u_{n} \rightarrow u$ in $C([0,1], H)$. Then 


$$
\begin{aligned}
\left\|N u_{n}(t)-N u(t)\right\| \leq & (1-t) \int_{0}^{t} s\left\|f\left(s, p\left(s, u_{n}(s)\right)\right)-f(s, p(s, u(s)))\right\| d s \\
& +t \int_{t}^{1}(1-s)\left\|f\left(s, p\left(s, u_{n}(s)\right)\right)-f(s, p(s, u(s)))\right\| d s \\
& \leq \int_{0}^{t}(1-s) s\left\|f\left(s, p\left(s, u_{n}(s)\right)\right)-f(s, p(s, u(s)))\right\| d s \\
& +\int_{t}^{1}(1-s) s\left\|f\left(s, p\left(s, u_{n}(s)\right)\right)-f(s, p(s, u(s)))\right\| d s \\
& =\int_{0}^{1}(1-s) s\left\|f\left(s, p\left(s, u_{n}(s)\right)\right)-f(s, p(s, u(s)))\right\| d s .
\end{aligned}
$$

So $N: C([0,1], H) \rightarrow C([0,1], H)$ is continuous. Now let $\Omega \subseteq C([0,1], H)$ be bounded i.e., there exists $r>0$ with $\|u(s)\| \leq r$ for all $s \in[0,1]$ and $u \in \Omega$. There exists $h_{r}$ as in the definition of $L_{l o c}^{1}$-Carathéodory with

$$
\|f(s, u)\| \leq h_{r}(s) \text { for a.e. } s \in[0,1] \text { and all }\|u\| \leq r \text {. }
$$

Now $N \Omega$ is bounded since for $t \in[0,1]$ and $u \in \Omega$ we have

$$
\|N u(t)\| \leq(1-t) \int_{0}^{t} s h_{r}(s) d s+t \int_{t}^{1}(1-s) h_{r}(s) d s \leq \int_{0}^{1} s(1-s) h_{r}(s) d s .
$$

Notice also for $u \in \Omega$ and $t \in[0,1]$ that

so we have

$$
(N u)^{\prime}(t)=\int_{0}^{t} s f(s, u(s)) d s+\int_{t}^{1}(1-s) f(s, u(s)) d s
$$

$$
\left\|(N u)^{\prime}(t)\right\| \leq \int_{0}^{t} h_{r}(s) d s+\int_{t}^{1}(1-s) h_{r}(s) d s \equiv \tau_{r}(t) .
$$

It is easy to check since $\int_{0}^{1} s(1-s) h_{r}(s) d s<\infty$ that $\tau_{r} \in L^{1}[0,1]$. Consequently $N \Omega$ is equicontinuous on $[0,1]$. Next we show

For $t \in[0,1]$, we have

$$
\alpha(N \Omega) \leq 2 \gamma \alpha(\Omega)
$$

$$
\alpha\left(N \Omega(t)=\alpha\left(\left\{\int_{0}^{1} K(t, s, p(s, u(s))) d s: u \in \Omega\right\}\right)\right.
$$




$$
\begin{aligned}
& \leq \alpha(\{K(t, s, p(s, u(s))): u \in \Omega, s \in[0,1]\}) \\
& \leq \alpha(K([0,1] \times[0,1] \times \overline{c o}(\Omega[0,1] \cup v[0,1])))
\end{aligned}
$$

since if $u \in \Omega$ and $s \in[0,1]$ we have

$$
p(s, u(s))=\lambda_{s} u(s)+\left(1-\lambda_{s}\right) v(s) \in \overline{c o}(\Omega[0,1] \cup v[0,1])
$$

where

$$
\lambda_{s}=\min \left\{1, \frac{M(s)}{\|u(s)-v(s)\|}\right\} .
$$

Thus

and so

$$
\begin{gathered}
\alpha(N \Omega(t)) \leq \gamma \alpha(\overline{c o}(\Omega[0,1] \cup v[0,1]))=\gamma \alpha(\Omega[0,1] \cup v[0,1]) \\
=\gamma \alpha(\Omega[0,1]) \leq 2 \gamma \alpha(\Omega)
\end{gathered}
$$

$$
\alpha(N \Omega)=\sup _{t \in[0,1]} \alpha(N \Omega(t)) \leq 2 \gamma \alpha(\Omega) .
$$

Thus (3.17) is true. Theorem 1.2 implies that (3.15) has a solution $y \in C([0,1], H)$. Next we claim $\|y(t)-v(t)\| \leq M(t)$ for $t \in[0,1]$. If the claim is true then $y$ is a solution of (3.9) and consequently $y$ is a solution of (3.8).

It remains to prove the claim. If the claim is not true then

$$
\|y(t)-v(t)\|-M(t)
$$

has its positive (absolute) maximum at, say, $t_{2} \in(0,1)$. Choose $t_{0}>0, t_{1}<1, t_{0}<$ $t_{2}<t_{1}$ with $\|y(t)-v(t)\|-M(t)>0$ for $t \in\left(t_{0}, t_{1}\right)$ and

$$
\left\|y\left(t_{1}\right)-v\left(t_{1}\right)\right\|-M\left(t_{1}\right)<\left\|y\left(t_{2}\right)-v\left(t_{2}\right)\right\|-M\left(t_{2}\right)
$$

this is possible since $\|y(1)-v(1)\|-M(1) \leq 0$. Also we have

$$
(\|y-v\|-M)^{\prime}\left(t_{2}\right)=0 .
$$

In addition for a.e. $t \in\left(t_{0}, t_{1}\right)$ we have

$$
\begin{aligned}
& \|y(t)-v(t)\|^{\prime \prime} \\
& \begin{aligned}
&\left.=\frac{\left\langle y(t)-v(t), y^{\prime \prime}(t)\right.}{\|y(t)-v(t)\|}-v^{\prime \prime}(t)\right\rangle+\left\|y^{\prime}(t)-v^{\prime}(t)\right\|^{2} \\
& \quad \frac{\left\langle\left\langle y(t)-v(t), y^{\prime \prime}(t)-v^{\prime \prime}(t)\right\rangle\right.}{\|y(t)-v(t)\|} \\
&=\frac{\left\langle y(t)-v(t),-f(t, p(t, y(t)))-v^{\prime \prime}(t)\right\rangle}{\|y(t)-v(t)\|^{3}} \\
& \geq M^{\prime \prime}(t) .
\end{aligned}
\end{aligned}
$$


To see the last inequality there are two cases to consider, namely $M(t) \neq 0$ and $M(t)=0$. If $M(t) \neq 0$ we have

$$
\begin{aligned}
& \frac{\left\langle y(t)-v(t),-f(t, p(t, y(t)))-v^{\prime \prime}(t)\right\rangle}{\|y(t)-v(t)\|} \\
&=\frac{\left\langle p(t, y(t))-v(t),-f(t, p(t, y(t)))-v^{\prime \prime}(t)\right\rangle}{M(t)} \geq M^{\prime \prime}(t)
\end{aligned}
$$

by (3.12), whereas if $M(t)=0$ we have

$$
\frac{\left\langle y(t)-v(t),-f(t, p(t, y(t)))-v^{\prime \prime}(t)\right\rangle}{\|y(t)-v(t)\|}=\frac{\left\langle y(t)-v(t),-f(t, v(t))-v^{\prime \prime}(t)\right\rangle}{\|y(t)-v(t)\|} \geq M^{\prime \prime}(t)
$$

by (3.13). Consequently,

$$
(\|y-v\|-M)^{\prime \prime}(t) \geq 0 \text { for a.e. } t \in\left(t_{0}, t_{1}\right) .
$$

Now (3.19) and (3.20) imply

$$
(\|y-v\|-M)^{\prime}(t) \geq 0 \text { for } t \in\left(t_{2}, t_{1}\right)
$$

and consequently

$$
\left\|y\left(t_{1}\right)-v\left(t_{1}\right)\right\|-M\left(t_{1}\right) \geq\left\|y\left(t_{2}\right)-v\left(t_{2}\right)\right\|-M\left(t_{2}\right) .
$$

This contradicts (3.18). Thus our claim is true and we are finished.

Remark: Let $H=\mathbf{R}$ and suppose $\alpha, \beta \in A C([0,1], \mathbf{R}) \cap C^{1}([0,1], \mathbf{R})$ with $\alpha^{\prime}, \beta^{\prime} \in$ $A C_{\text {loc }}((0,1), \mathbf{R})$, are respectively lower and upper solutions of (3.8) (i.e., $\alpha^{\prime \prime}+$ $f(t, \alpha) \geq 0$ a.e. on $[0,1], \alpha(0) \leq 0, \alpha(1) \leq 0$ and $\beta^{\prime \prime}+f(t, \beta) \leq 0$ a.e. on $[0,1]$, $\beta(0) \geq 0, \beta(1) \geq 0)$ with $\alpha(t) \leq \beta(t)$ for $t \in[0,1]$. Its easy to check that

$$
v=\frac{\alpha+\beta}{2} \text { and } M=\frac{\beta-\alpha}{2}
$$

satisfy (3.12), (3.13) and (3.14) in this case. Of course, (3.10) is satisfied with $\gamma=0$. Consequently a special case of Theorem 3.3 is the result in $[3,10]$.

\section{References}

[1] Brezis, H. and Browder, F.E., Existence theorems for nonlinear integral equations of Hammerstein type, Bull. Amer. Math. Soc. 81 (1975), 73-78.

[2] Dugundji, J. and Granas, A., Fixed point theory, Monografie Mat., PWN Warsaw 1982.

[3] De Coster, C., Grossinho, M.R. and Habets, P., On pairs of positive solutions for a singular boundary value problem, Appl. Anal. 59 (1995), 241-256.

[4] Frigon, M., Boundary and periodic problems for systems of nonlinear second order differential equations, Topol. Methods Nonlinear Anal. 1 (1993), 259-274.

[5] Frigon, M. and O'Regan, D., Existence results for initial value problems in Banach spaces, Differential Eqns. Dyn. Systems 2 (1994), 41-48. 
[6] Guenther, R.B. and Lee, J.W., Some existence results for nonlinear integral equations via topological transversality, J. Int. Eqns. Appl. 3 (1993), 195-209.

[7] Lakshmikantham, V. and Leela, S., Nonlinear Differential Equations in Abstract Spaces, Pergamon Press, New York 1981.

[8] Lee, J.W. and O'Regan, D., Existence principles for nonlinear integral equations on semi-infinite and half open intervals, In: Advances in Nonlinear Dynamics (ed. by S. Sivasundaram and A.A. Martynyuk), Gordon and Breach Science Publishers, Ltd., Amsterdam 1997, 355-364.

[9] Martin, R.H., Nonlinear Operators and Differential Equations in Banach Spaces, John Wiley and Sons, New York 1976.

[10] O'Regan, D., Existence principles and theory for singular Dirichlet boundary value problems, Diff. Eqns. and Dynamical Systems 3 (1995), 289-304.

[11] O'Regan, D., Existence results for nonlinear internal equations, J. Math. Anal. Appl. 192 (1995), 705-726.

[12] O'Regan, D., Existence principles for nonlinear operator equations, Boundary Value Problems for Functional Differential Equations (ed. by J. Henderson), World Scientific Press, River Edge, New Jersey (1995), 251-260.

[13] O'Regan, D., Integral inclusions of upper semi-continuous or lower semicontinuous type, Proc. Amer. Math. Soc. 124 (1996), 2391-2399.

[14] O'Regan, D., Singular Dirichlet boundary value problems I: Superlinear and nonresonance case, Nonlinear Analysis 29 (1997), 221-245.

[15] Vaughn, R., Existence and comparison results for nonlinear Volterra integral equations in a Banach space, Appl. Anal. 7 (1978), 337-348.

[16] Yosida, K., Functional Analysis, Springer Verlag, Berlin 1978. 


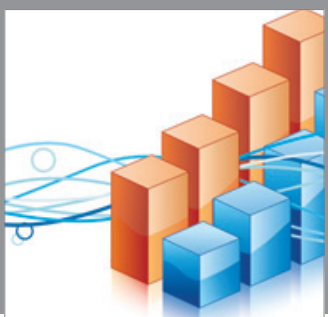

Advances in

Operations Research

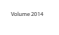

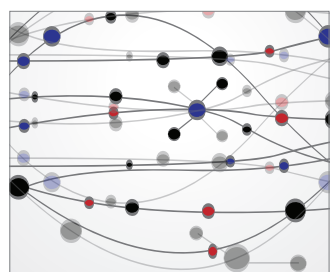

\section{The Scientific} World Journal
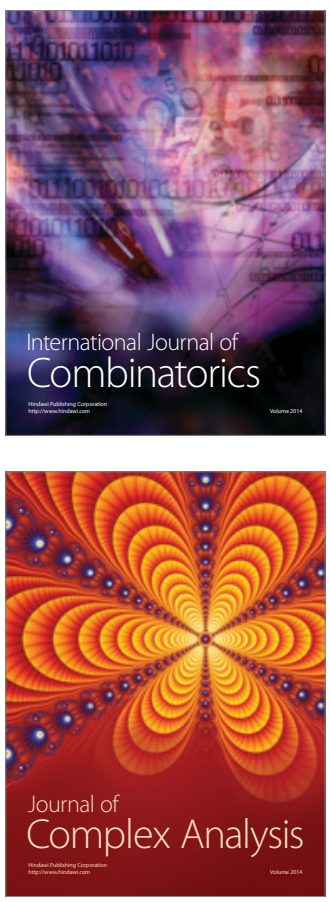

International Journal of

Mathematics and

Mathematical

Sciences
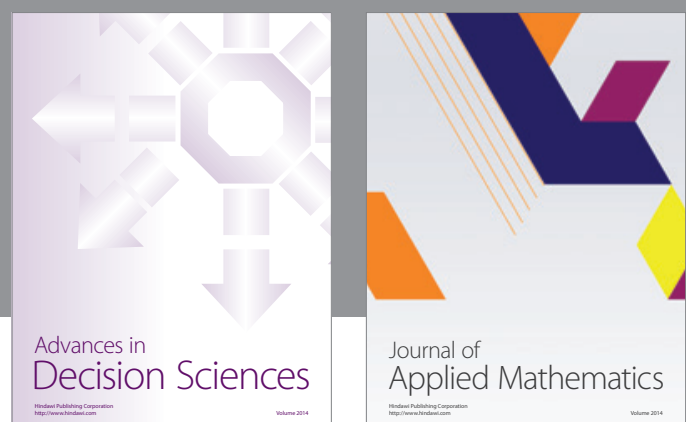

Journal of

Applied Mathematics
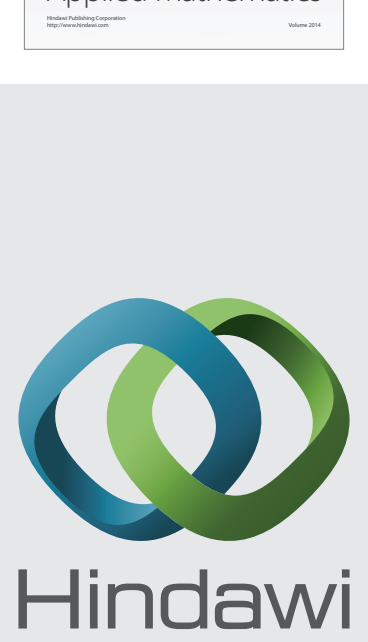

Submit your manuscripts at http://www.hindawi.com
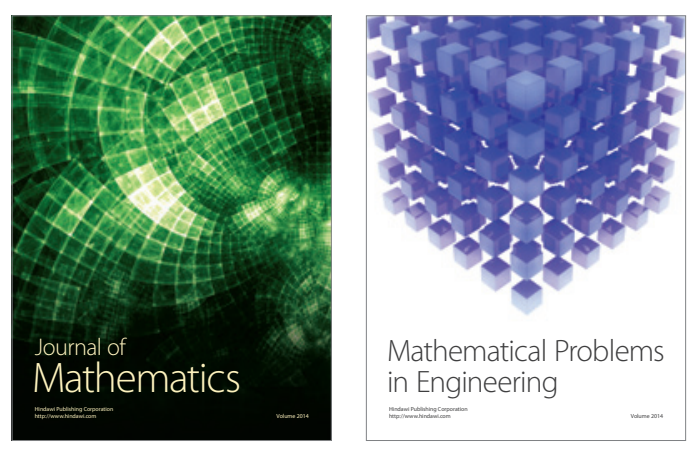

Mathematical Problems in Engineering
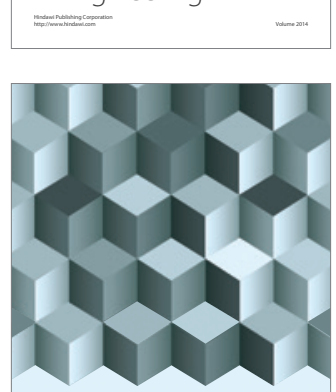

Journal of

Function Spaces
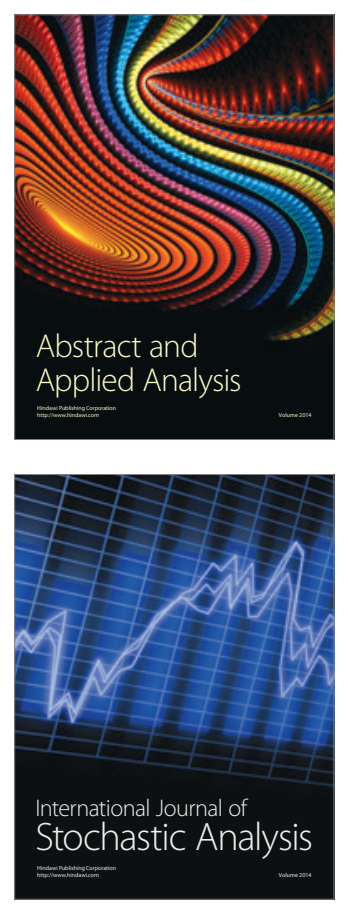

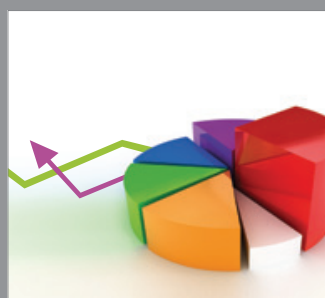

ournal of

Probability and Statistics

Promensencen
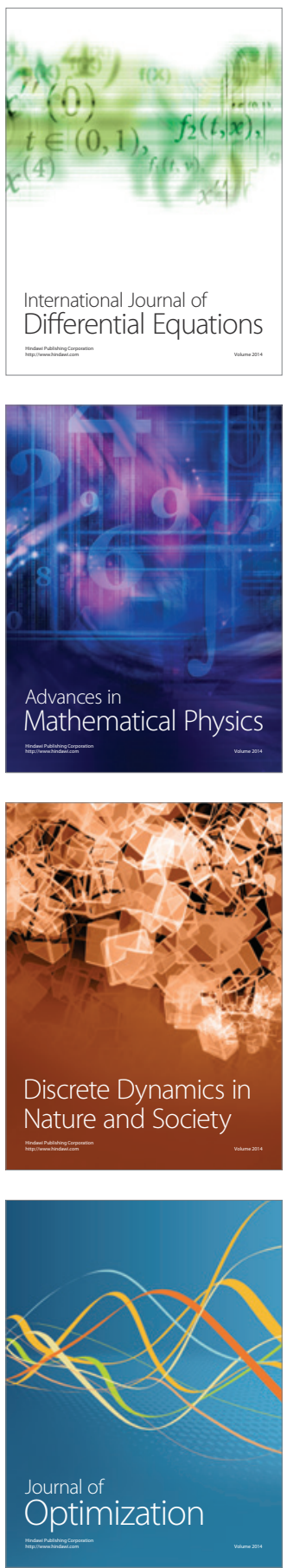